\title{
Praxisparalleles Gewerbe: orthomolekulare Ernährungsmedizin
}

— Die orthomolekulare Ernährungsmedizin ist ein wichtiger Baustein der Schulmedizin, denn durch die gezielte Versorgung mit Mikronährstoffen können die Gesundheit unterstützt und Krankheiten Therapie-begleitend behandelt werden. Die Relevanz von Mikronährstoffen für die ernährungsmedizinische Therapie belegen internationale Studien. Für Ärzte kann daher das praxisparallele Gewerbe mit orthomolekularer Ernährungsmedizin eine sinnvolle Option sein, das therapeutische Spektrum ihrer Praxis auszuweiten. Die Arzt-exklusive Abgabe orthomolekularer Produkte dient zum einen der Patientenbindung, zum anderen kann sich die Praxis dadurch von anderen abgrenzen. Nicht zuletzt ist auch der wirtschaftliche Nutzen eines solchen praxisparallelen Gewerbes erwähnenswert. Die Orthomed GmbH unterstützt Ärzte bei der Gründung -
z.B. mit kostenlosen Gründungsseminaren, die deutschlandweit stattfinden und unter anderem über juristische Aspekte informieren. Außerdem bietet das Unternehmen individuelle Coachings und Fortbildungsveranstaltungen sowie Unterstützung für das Praxis-Marketing an. Eine Vertragsbindung besteht für die Arztpraxen nicht.

Die Orthomed GmbH hat über 20 orthomolekulare Produkte mit breitem Indikationsspektrum in seinem Portfolio (www.orthomedgmbh.de). Darunter orthofertil ${ }^{\circledR}$ plus (bei Fertilitätsstörungen des Mannes), orthocare ${ }^{\circledast}$ $\mathrm{m}$ (bei Prostatakrebs) oder orthonorm ${ }^{\circledR} \mathrm{m}$ (bei Männern mit chronischer Erschöpfung, Burn-out-Syndrom und stressbedingten Erkrankungen mit metabolischer Störung).

Nach Informationen der

Orthomed $\mathrm{GmbH}$, Langenfeld

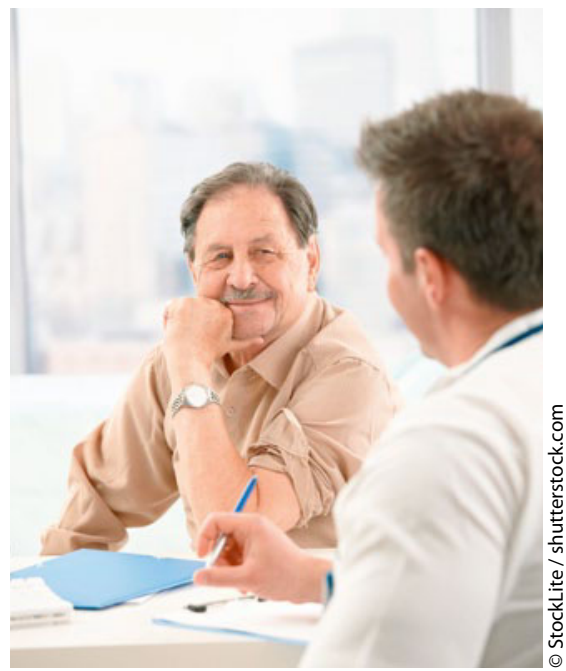

Orthomolekulare Produkte in der Artzpraxis erwerben - ein neuer Service für Patienten.

\section{Pflanzliche Therapie von Harnwegsinfekten - klinisch geprüfte Produkte bevorzugen}

_ Um der wachsenden Bedrohung durch Antibiotikaresistenzen entgegenzuwirken, ist es sinnvoll, bei unkomplizierten viralen und bakteriellen Infektionen auf pflanzliche Präparate auszuweichen. Allerdings sollte die Wirksamkeit der eingesetzten pflanzlichen Präparate belegt sein:„,Um eine vergleichbare Wirkung zu erzielen, sollte dabei klinisch geprüften Produkten der Vorzug gegeben werden", erläutert Prof. Dr. Uwe Frank vom
Universitätsklinikum Freiburg. Dies trifft beispielsweise auf die Senföle aus Kapuzinerkresse und Meerrettichwurzel (ANGOCIN ${ }^{\circledR}$ Anti-Infekt N) zu: Bei akuter Sinusitis, akuter Bronchitis und akuter Blasenentzündung belegen prospektiven Kohortenstudien an Erwachsenen und Kindern, dass die pflanzliche Alternative vergleichbar wirksam ist wie chemische Standard-Antibiotika bei einem erheblich besseren Verträglichkeits- und Si-

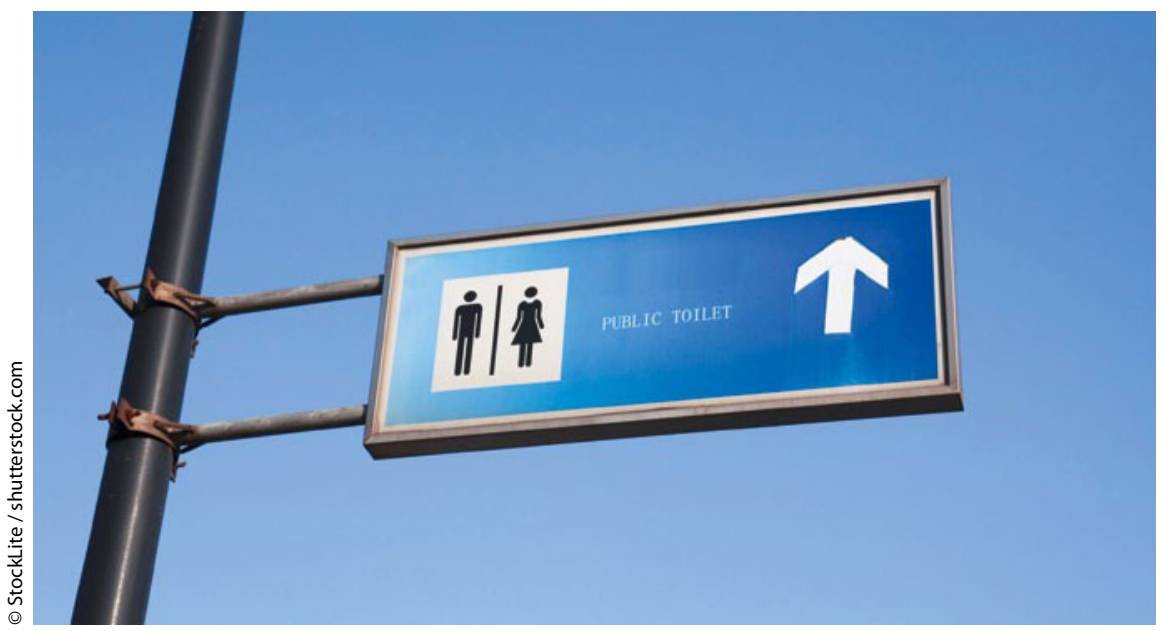

Harnwegsinfekt? Klinisch geprüfte Pflanzen-Präparate sind eine Alternative zu Antibiotika. cherheitsprofil des pflanzlichen Präparates. Eine Resistenzentwicklung wurde bisher ebenfalls nicht beobachtet. Bei vielen anderen pflanzlichen Präparaten, wie zum Beispiel den Cranberrys, fehlt dieser Nachweis der Wirksamkeit. Cranberrys wurden bereits in früheren Studien in der Prophylaxe von Harnwegsinfekten untersucht, der entsprechende Wirksamkeitsnachweis konnte jedoch nicht erbracht werden. Auch eine neue Studie belegt: Im Vergleich mit Placebo konnte keine prophylaktische Wirkung von Cranberrys bei rezidivierenden Harnwegsinfekten festgestellt werden. Es gibt demzufolge in Deutschland kein zugelassenes Arzneimittel mit diesem Wirkstoff. Alle Cranberry-Produkte, die vermarktet werden, sind Nahrungsergänzungsmittel, die keine Zulassung benötigen und keinen Qualitätsnachweis erbringen müssen. Das hat die europäische Sicherheitsbehörde für Lebensmittel (EFSA) mit der Ablehnung eines für Cranberrys angemeldeten "health-claims" noch einmal deutlich gemacht.

Verschiedene In-vitro-Studien zeigen, dass die Senföle gegen 13 klinisch relevante Bakterienarten eine ausgeprägte keimhemmende Wirkung besitzen, sogar gegen den Problemkeim MRSA.

Nach Informationen von

Repha, Langenhagen 